\title{
Actions speak louder: Young female patients with acute ischemic stroke in the emergency department
}

\author{
Mor Saban, ${ }^{1}$ Tal Shachar, ${ }^{2}$ Heli Patito, ${ }^{1}$ Lev Zaretsky ${ }^{1}$ \\ ${ }^{1}$ Department of Nursing, Faculty of Health and Welfare Sciences, University of Haifa, Haifa; ${ }^{2}$ Faculty of \\ Medicine, Bar-Ilan University, Safed, Israel
}

\begin{abstract}
The aim was to explore the diagnostic cascade of young females with acute ischemic stroke (AIS) in the emergency department (ED) setting. A retrospective case series study was conducted between the years 2016-2018 in the ED of a tertiary hospital $(\mathrm{N}=10)$. We collected socio-demographic data, clinical risk factors and co-morbidities, ED characteristics and medical examination related data. Ten females presenting with AIS were identified. Results show that each case had a variety of characteristics, there are no similar medical background or clear-cut risk factors, and each case has been presented clinically different. All these factors, with the possible added effect of age and sex bias serve as possible hindrance for correct and efficient diagnosis of stroke in young females. In conclusion, clinical presentation of young female with AIS is misleading. Initial examination in the ED setting may appear to be the determining point of impact on the outcome severity in young females.
\end{abstract}

Correspondence: Mor Saban, Department of Nursing, Faculty of Health and Welfare Sciences, University of Haifa, Haifa, Israel.

Tel.: +972/0.203.0191.

E-mail: morsab1608@gmail.com

Key words: Cerebrovascular accident; Emergency department; Ischemic stroke; Young female.

Contributions: all of the authors interpreted the data, edited and approved the final article. MS, TS, LZ and HP drafted and conceived the study. MS, LZ and HP analyzed the data, designed the study and performed data collection. MS and TS take responsibility for the paper as a whole. All of the authors attest to meet the ICMJE.org authorship criteria.

Conflict of interest: the authors declare no potential conflict of interest.

Funding: none.

Received for publication: 23 May 2019

Revision received: 8 July 2019.

Accepted for publication: 8 August 2019.

This work is licensed under a Creative Commons Attribution 4.0 License (by-nc 4.0).

๑C Copyright: the Author(s), 2019

Licensee PAGEPress, Italy

Emergency Care Journal 2019; 15:8300

doi:10.4081/ecj.2019.8300

\section{Introduction}

Stroke in young females is an under-researched group with an increasing number of occurrences. ${ }^{1-3}$ Few statistical and epidemiological studies have been published regarding this subject. ${ }^{1,45}$ Yet, none of these studies have shed light on emergency department (ED) management and the critical time lags influence on the diagnosis of stroke in young females. A large scale meta-analysis confirms that females generally experience poorer function outcome and lower quality of life after stroke in comparison to men. ${ }^{2}$ In the $\mathrm{ED}$, the probability of misdiagnosis of acute ischemic stroke (AIS) is high, and up to $30 \%$ of suspected stroke presentations have a different diagnosis. ${ }^{6-8}$ In addition, females have increased probability of a stroke mimic, delays in acute imaging, and a lower likelihood of receiving treatment, due to unusual presentation of symptoms and timely recognition. ${ }^{1}$ Data on females shows that each year approximately 55,000 more females than males experience stroke, females have a higher lifetime risk of stroke in comparison with males and the frequency of poor outcomes is higher in females. ${ }^{4,9,10}$

Despite preexisting knowledge regarding female risk factors and presentation in stroke, there are no prior studies examining the ED management and critical time lags influencing the diagnosis of AIS in young females. The purpose of this case series is to explore the diagnosis and treatment cascade in young females presenting with AIS to the ED.

\section{Case Reports}

During the study, 10 young female patients suspected and diagnosed with AIS were included. The patients' clinical characteristics are summarized in Table 1.

The average age is 41.5 years old (range 28-55), eight of them were born in Israel, seven are of Jewish nationality, nine are married, and eight of the patients have between one and seven children. Clinical risk factors such as diabetes mellitus, hypertension, atrial fibrillation (AF), prior stroke, valvular disease and psychiatric disorders were presented in some of the cases without a clear mutual relation among the patients (cases 1, 2, 3, 5, 7, 8, 9 and 10). Mode of arrival to the ED was relatively conclusive, nine females arrived by emergency medical services (EMS) and only one arrived independently (case 2). All patients except two (cases 4 and 10) were highly prioritized according to Canadian triage and acuity scale, with a level of 1 . Seven patients were primarily examined by a neurologist (cases 1, 2, 3, 5, 6, 7 and 8), whereas the other three were examined by an internist, gynecologist, psychiatrist, followed by a neurological assessment (cases 3,9 and 10). National institute health stroke scale (NIHSS) determined by the neurologists was high in all cases $(13.9 \pm 11.05)$. However, the range was heterogeneous (1-42). Time delay from stroke onset to ED arrival was 
$148 \pm 84.54$ minutes, whereas ED to computed tomography (CT) time was $98 \pm 196.95$ minutes. In regard to treatment type, two patients received intra venous tissue plasminogen activator (IV. tPA) (cases 5 and 9), six patients undergoing mechanical embolus removal in cerebral ischemia (MERCI) (cases 1, 3, 4, 7, 8 and 10) while the remaining received combined treatment (cases 2 and 6 ).

Medical examinations during hospitalization showed new findings in several females. Trans esophageal echo (TEE) examination demonstrated mild mitral regurgitation found in two cases (cases 2 and 5) and patent foramen ovale (PFO) found in three cases (cases 4, 6 and 9). In addition, elevated levels of thyroid stimulating hormone (cases 3 and 8 ) as well as dyslipidemia in some of the females (cases 3 and 5) were found. In regard to hospitalization outcomes, majority of patients did not suffer any complications whereas two patients showed aspiration pneumonia and presented with cerebral hemorrhage (cases 3,6 and 9). Length of stay was $43.5( \pm 76.59)$ days. Most females were discharged to rehabilitation (cases 1, 3, 5, 6, 7, 8 and 10) while the remaining were discharged home with average modified Rankin scale (mRS) score of 3 (Table 2).

\section{Illustrative cases}

\section{Case \#6}

A 44-year-old female, mother of five, arrived to the ED by an EMS team. The team reported that the patient was found on the ground with decreased level of consciousness after preforming physical exercise one hour prior to the team arrival. On admission, clinical presentation included combined neurological symptoms with NIHSS 10. A history of osteoarthritis without medication use. Fast-neurological evaluation was performed and the patient was sent to CT, demonstrating complete obstruction of right M1, as well as impression of A1 segmental filling problem of the same side without distal anterior cerebral artery filling. Computed tomography angiography showed close to a complete obstruction. In light of these findings tPA was administered followed by MERCI under general anesthesia due to massive vomiting. Time from CT to groin puncture was 64 minutes. During MERCI, four unsuccessful attempts were conducted to clear the occlusion. During TEE examination PFO was found. In addition, two decompression craniotomies were performed in light of repeated herniation. Several complications were documented - aspiration pneumonia, urinary tract infection and nosocomial infections. Following 271 days of hospitalization, patient was discharged for rehabilitation.

\section{Case \#9}

A 39-year-old female, with a history of migraine without a history of medication use, arrived to the ED of a secondary hospital during evening time with a pounding headache and a nonspecific weakness complaint. Immediate neurological evaluation was performed, followed by a CT examination and administration of tPA. Patient claimed to improve after treatment and upon her request was discharged home. A few hours later, felt unwell and was transported to a tertiary hospital by an EMS team. New neurological evaluation was performed by a neurologist that concluded a mental component was present. Due to the patient behavior a referral for psychiatric evaluation was made, which showed no findings. CT examination was performed with signs of occlusion. As such, admitted for observation. During night of admission patient status deteriorated, presenting with irritability and side complains. On the following morning an MRI was conducted, showing a massive hemispheric infarct. Due to these findings the patient was not eligible for a MERCI. Extensive evaluation was made during hospi- talization including TEE, demonstrating a large PFO. All other examination appeared normal and the patient was discharge home with mRS 1.

\section{Case \#10}

A 28-year-old female, with history of epilepsy, bipolar disorder, discopathy, heavy smoking and a long-standing use of cannabis. Medication history included lithium, oxazepam, carbamazepine and an intrauterine device. Patient was brought to the urgent area by an EMS team presenting postictal phase, remained unconscious for several hours and, due to patient history, a psychiatric evaluation was requested. After additional hours without improvement in consciousness followed by neurological deterioration the patient was admitted to the resuscitation bay and fast tract protocol has been activated. Findings showed extensive basilar infarction prompting patient transport for MERCI (CT to groin time was 131 minutes) where the clot was successfully extracted. Yet, extensive brainstem damage remains.

\section{Discussion}

Stroke related risk factors and their impact on young adults has been demonstrated in several publications. ${ }^{11,12}$ Yet, to the best of our knowledge, the challenge of diagnosing AIS in young females has not been addressed in relation to clinical presentation and patient outcome at the ED. Detecting and diagnosing AIS is a major challenge for medicine in light of the importance of fast treatment. ${ }^{6,11}$ Of the cases we reviewed, we found that the challenge is even greater and significant when it comes to young females.

Acceptance that delay in presentation, evaluation, diagnosis and treatment of females with AIS may contribute to the association between female sex and more severe stroke is present. ${ }^{13}$ One important finding is that the vast majority of females arrived by EMS, which means that the patients and their families perceived the event as an urgent and life-threatening situation. As such, these females were classified as high priority at triage, were received and treated in the resuscitation bay. Yet, time for CT was longer than recommended ( $\leq 25 \mathrm{~min}$ ). ${ }^{14}$ In addition, cases 4,9 and 10 underwent primary evaluation by either a gynecologist, psychiatrist or internist physician, followed by a neurological examination. Paradoxically, there is a supposed phenomenon in which the diagnostic stage is delayed despite a rapid prehospital and triage stage. When viewing the process as a whole, it appears that preliminary diagnostic stages are the point of bottleneck formation. We offer two possible explanations for these paradoxical occurrences.

First, heterogenic clinical features in distinct ED applicants such as females present occasionally with atypical symptoms. ${ }^{15}$ The high range of NIHSS scores in our cases can be explained by current literature. In fact, limited evidence suggests that females with AIS present different symptoms than men, as it is known for acute coronary syndrome. ${ }^{16-18}$ Many of the factors that could cause worse stroke-related outcomes in females, result from delays in arrival or delays in acute treatment after stroke. ${ }^{13,19}$ Other studies have reported impaired levels of consciousness among females more frequently than men, which could be a contributing factor for increased stroke severity. ${ }^{16}$ Second, potential age and sex related biases, in line with current literature risk factors may lead to a gap in patient-personnel communication. Some studies of gender differences in the management of AIS have shown that females are investigated less thoroughly. ${ }^{19}$ In a study conducted in 2016 results showed that sex-based stroke treatment disparity was noted among 


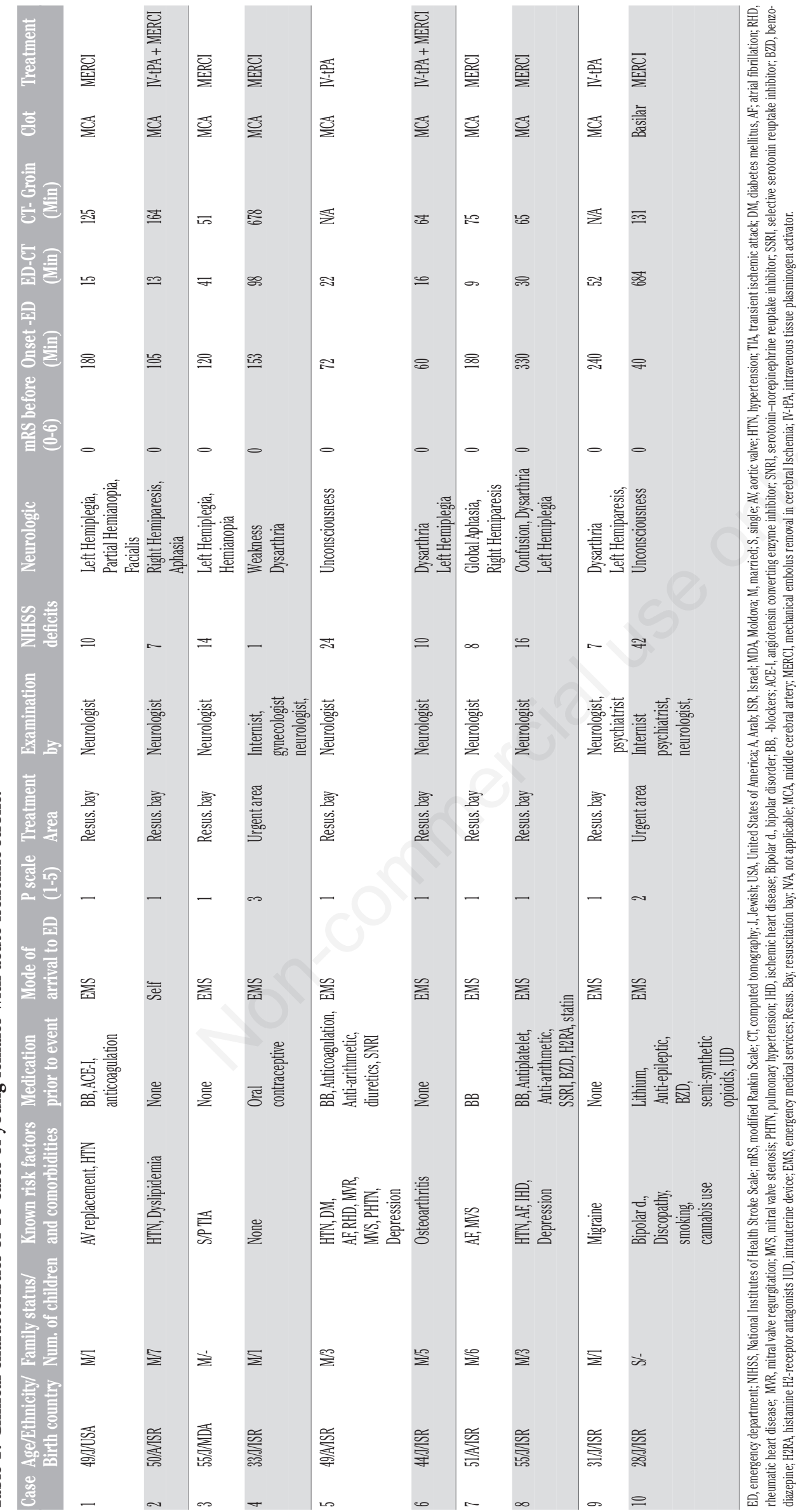


Table 2. Outcomes hospitalization of the young females.

\begin{tabular}{|c|c|c|c|c|c|}
\hline Case & Medical examinations in hospitalization (New finding) & Complications & LOS (day) & mRS at discharge (0-6) & Discharge to \\
\hline 1 & Low INR level -Coumadin untherapeutic & None & 12 & 2 & Rehabilitation \\
\hline 2 & Holter 24 h-VPBS/APBS, TEE: Mild MR & None & 5 & 1 & Home \\
\hline 3 & Holter 24 h- AF, High TSH, Dyslipidemia & Hemorrhage, aspiration pneumonia & 32 & 4 & Rehabilitation \\
\hline 4 & TEE: PFO, DUS: DVT & None & 9 & 1 & Home \\
\hline 5 & TEE: Mild MR, Dyslipidemia & None & 11 & 5 & Rehabilitation \\
\hline 6 & TEE: PFO & Hemorrhage, aspiration pneumonia & 270 & 5 & Rehabilitation \\
\hline 7 & AF untreated & None & 6 & 2 & Rehabilitation \\
\hline 8 & Holter 24 h-VPBS/APBS, High TSH & None & 37 & 3 & Rehabilitation \\
\hline 9 & TEE: PFO & None & 11 & 1 & Home \\
\hline 10 & TEE-Tricuspid valve regurgitation & Hemorrhage & 42 & 5 & Rehabilitation \\
\hline
\end{tabular}

LOS, length of stay; mRS, modified Rankin Scale; INR, international normalized ratio; VPBS, ventricular premature beats; APBS, atrial premature beats; TEE, transesophageal echocardiography; MR, mitral regurgitation; AF, atrial fibrillation; TSH, thyroid stimulating hormone; PF0, patent foramen ovale; DUS, duplex ultrasound; DVT, deep vein thrombosis.

different ethnicities. Yet, these study findings emphasize that adherence to a stroke performance program not only improves care but can also resolve disparity. ${ }^{18}$

During medical examination, PFO has been found in three cases $(4,6$, and 9). Several publications have shown high prevalence of PFO in a population of young AIS patients. ${ }^{10}$ While it has been found that closure of PFO is strongly associated with a lower risk of recurrent stroke, there is little evidence of it being a direct cause. ${ }^{18}$ Stroke related cardiac risk factors such as AF, valvular malformation and ischemic heart disease, were present in four of the cases we examined (1, 5, 7 and 8). Sex differences in clinical features and management of AF suggest that female subjects are more symptomatic relative to men. ${ }^{12,13}$ In regard to stroke, sex differences are, in part, explained by differences in pre-stroke characteristics and clinical presentation. Additionally, delayed diagnosis might be explained by the presence of psychiatric history and/or psychiatric evaluation as can be seen in cases 5,8 and 10. This is in accordance to the presence of psychiatric causes in the differential diagnosis of acute neurological deficits. ${ }^{16}$ However, viewing the cases we presented, there are no consistent patterns of hospitalization examination or treatment. While stroke protocol was indeed activated in all female patients, time lags leading to activation showed remarkable variations.

\section{Conclusions}

Young female AIS patients are associated with an atypical presentation that leads to delayed diagnosis and fatal outcomes in the ED. Two factors may be the reason for this delay. First, affiliation to the female gender and second, presentation in an early age. These factors are associated in medical literature with delayed diagnosis and treatment. As presented, several females were labeled as having a psychiatric event leading to time consuming consultations and thus delayed diagnosis.

To the best of our knowledge, no other study has examined the complexity of early diagnosis of AIS in young female patients in the ED. Thus, further researches with a larger sample size are needed in order to explore diagnosis and treatment variables affecting young females with suspected AIS. Stroke researchers must consider the translational relevance of such sex differences as many are unaware of the potential confounding factors of sex differences. A greater understanding of the mechanisms underlying sex difference in stroke and responsiveness to neuroprotection will lead to more appropriate treatment strategies for patients of both sexes. Additionally, recognition of potential gender differences in stroke symptoms through education of EMS and ED teams aimed at both the public and health care professionals, could result in decreased out-of-hospital and in-hospital delays. Thus, increasing access to acute stroke therapy in women. Increasing ED staff awareness and performing medical simulation of such case studies may lead to a decrease in delayed or misdiagnosed patients.

\section{References}

1. Bushnell C, McCullough LD, Awad IA, et al. Guidelines for the prevention of stroke in women: a statement for healthcare professionals from the American Heart Association/American Stroke Association. Stroke 2014;45:1545-88.

2. Newman-Toker DE, Moy E, Valente E, et al. Missed diagnosis of stroke in the emergency department: a cross-sectional analysis of a large population-based sample. Diagnosis 2014;1:15566.

3. Andrew NE, Srikanth V. Sex differences in stroke outcomes: A case for better health care for older women. Neurology 2018;90:995-6.

4. Nightingale AL, Farmer RD. Ischemic stroke in young women: a nested case-control study using the UK general practice research database. Stroke 2004;35:1574-8.

5. Kim JA. Book review: creative destruction of medicine: how the digital revolution will create better health care. Healthc Inform Res 2013;19:229-31.

6. Arch AE, Weisman DC, Coca S, et al. Missed ischemic stroke diagnosis in the emergency department by emergency medicine and neurology services. Stroke 2016;47:668-73.

7. Moy E, Barrett M, Coffey R, et al. Missed diagnoses of acute myocardial infarction in the emergency department: variation by patient and facility characteristics. Diagnosis 2015;2:29-40.

8. Barrett KM, Levine JM, Johnston KC. Diagnosis of stroke and stroke mimics in the emergency setting. CONTINUUM: Lifelong Learn Neurol 2008;14:13-27.

9. Kim JS, Lee KB, Roh H, et al. Gender differences in the functional recovery after acute stroke. J Clin Neurol 2010;6:183-8.

10. Powers WJ, Rabinstein AA, Ackerson T, et al. 2018 guidelines for the early management of patients with acute ischemic stroke: a guideline for healthcare professionals from the American Heart Association/American Stroke Association. 
Stroke 2018;49:e46-e99.

11. Kizer JR, Devereux RB. Patent foramen ovale in young adults with unexplained stroke. N Engl J Med 2005;353:2361-72.

12. Singh NN, Pan Y, Muengtaweeponsa S, et al. Cannabis-related stroke: case series and review of literature. J Stroke Cerebrovasc Dis 2012;21:555-60.

13. Barker-Collo S, Bennett DA, Krishnamurthi RV, et al. Sex differences in stroke incidence, prevalence, mortality and disability-adjusted life years: results from the Global Burden of Disease Study 2013. Neuroepidemiology 2015;45:203-14.

14. Sabetay S, Hochman O, River Y. Four-year follow-up of thrombolysis treatment improvement following the implementation of the 25-minute door-to-CT protocol of the Israeli Ministry of Health. J Neurol Sci 2017;381:435.

15. Saban M, Zaretsky L, Patito H, et al. Round-off decision-mak- ing: Why do triage nurses assign STEMI patients with an average priority? Int Emerg Nurs 2019;43:34-9.

16. Santalucia P, Pezzella FR, Sessa M, et al. Sex differences in clinical presentation, severity and outcome of stroke: results from a hospital-based registry. Eur J Intern Med 2013;24:16771.

17. Haast RA, Gustafson DR, Kiliaan AJ. Sex differences in stroke. J Cereb Blood Flow Metab 2012;32:2100-7.

18. Asdaghi N, Romano JG, Wang K, et al. Sex disparities in ischemic stroke care: FL-PR CReSD study (Florida-Puerto Rico Collaboration to Reduce Stroke Disparities). Stroke 2016;47:2618-26.

19. Reid JM, Dai D, Gubitz GJ, et al. Gender differences in stroke examined in a 10-year cohort of patients admitted to a Canadian teaching hospital. Stroke 2008;39:1090-5. 